\title{
An Analysis of CS Algorithms Efficiency for Sparse Communication Signals Reconstruction
}

\author{
Radomir Mihajlović, Marijana Šćekić, Andjela Draganić, Srdjan Stanković \\ Faculty of Electrical Engineering \\ University of Montenegro \\ Podgorica, Montenegro \\ e-mail: andjelad@ac.me
}

\begin{abstract}
As need for increasing the speed and accuracy of the real applications is constantly growing, the new algorithms and methods for signal processing are intensively developing. Traditional sampling approach based on Sampling theorem is, in many applications, inefficient because of production a large number of signal samples. Generally, small number of significant information is presented within the signal compared to its length. Therefore, the Compressive Sensing method is developed as an alternative sampling strategy. This method provides efficient signal processing and reconstruction, without need for collecting all of the signal samples. Signal is sampled in a random way, with number of acquired samples significantly smaller than the signal length. In this paper, the comparison of the several algorithms for Compressive Sensing reconstruction is presented. The one dimensional band-limited signals that appear in wireless communications are observed and the performance of the algorithms in non-noisy and noisy environments is tested. Reconstruction errors and execution times are compared between different algorithms, as well.
\end{abstract}

Keywords-basis pursuit, Compressive Sensing, iterative hard thresholding, orthogonal matching pursuit, wireless signals

\section{INTRODUCTION}

Compressive Sensing (CS) [1]-[9] appears as a new paradigm that recently has attracted the attention of many researches in the signal processing community and applied mathematics. It provides signal sampling at the rates that are far below Nyquists. Therefore, CS provides memory saving and shortens the execution time, which is of great importance in many applications. Besides lowering the sampling rate, CS provides successful signal processing in the cases when the missing samples phenomenon occurs [1]. It has been applied in large number of areas, such as medicine, radars, communications, speech and image processing [5]-[10], etc.

CS is based on the complex mathematical algorithms for finding the sparse approximation of the signal. Therefore, in order to reconstruct signal with high accuracy, the signal has to be sparse in certain domain. Additionally, sampling procedure in the CS should be done in a way that provides efficient reconstruction with number of measurement as small as possible. There are many reconstruction algorithms, such as greedy strategies, $l_{1}$-minimization, etc. [5], [11]-[13] for finding the sparsest solution of the optimization problem. Another methodology for capturing regularity from the data is minimum description length (MDL) principle [14], [15]. MDL allows data compression using limited set of the observed data.

In this paper, we detail the application of different CS algorithms to the reconstruction of randomly sampled bandlimited signals that appear in communications. These are multicomponent signals that exhibit sparseness property in the frequency domain, and could be described as follows:

$$
x(n)=\sum_{k=1}^{K} a_{k} e^{-j 2 \pi k n}, n \in[0, N-1],
$$

where $K$ denotes number of sinusoidal components in the observed signal, while $N$ is the signal length. As these signals usually have very large bandwidth, i.e. large $N$, and small number of non-zero components in frequency domain $(K<<N)$, they are possible candidates for CS application. The application of several commonly used CS signal reconstruction solutions applied to these signals is presented in the paper. The performance in the noiseless and noisy signal case is examined. The comparison results and performance analysis are given, as well.

The paper is organized as follows. In Section II, the fundamental concepts of CS theory and signal reconstruction method are given. The overview of the applied CS algorithms is given in the Section III. The CS application to band-limited sparse signals is discussed in Section IV. Concluding remarks are given in Section V.

\section{COMPRESSIVE SENSING}

To be able to efficiently analyze the signals using the traditional methods, it would be desirable to deal with the full set of signal samples. Usually, this number of samples is very large. Another difficulty in signal analysis is presence of noise which causes missing information about the signal, and thus complicate signal analysis and further processing. Recently, a new method for signal reconstruction and processing, that can outperform these difficulties, is intensively studied. This method, called CS, provides efficient analysis of the signal even in the cases when there is no complete information about the signal. Certain requirements must be satisfied in order to

This work has been supported by the project CS-ICT (New ICT Compressive sensing based trends applied to: multimedia, biomedicine and communications), funded by Montenegrin Ministry of Science. 
$3^{\text {rd }}$ Mediterranean Conference on Embedded Computing

apply CS procedure. Namely, CS deals with the signals which are sparse in a certain transform domain, i.e., have concise representations when expressed in the proper basis. Signal shows sparsity in different domains, such as Fourier, discrete cosine transform, wavelet, time domain, etc. In general, a $K$ sparse signal in a specific domain can be completely characterized by $M$ measurements $(M>K)$ with $M<<N$, where $N$ is the number of samples imposed by the Shannon-Nyquist theorem and $K$ denotes the number of non-zero samples in certain domain.

The discrete-time signal $x$ of length $N$ can be represented in terms of basis vectors as follows [3]:

$$
\mathbf{x}=\sum_{i=1}^{N} z_{i} \psi_{i}=\psi \mathbf{z}
$$

where $z_{i}$ represents the transform domain coefficient, $\psi_{i}$ is a basis vector, $\boldsymbol{\psi}$ denotes $N \mathrm{x} N$ transform matrix. For signal $\mathbf{z}$ it is said that is $K$-sparse in the $\boldsymbol{\psi}$-domain. Signal measurements are acquired from the domain where signal have "dense" representation, where $M<<N$ holds. Some a priori defined conditions have to be satisfied in order to reconstruct signal from the acquired, small number of measurement. Firstly, the measurement matrix, denoted as $\phi$, must be incoherent with the basis matrix $\psi$. Lower coherence leads to a smaller number of measurements required to recover the entire signal. Second requirement is the signal sparsity in the certain domain.

If the measurement vector is denoted as $\boldsymbol{b}$, then the signal measuring procedure can be defined using the measurement matrix $\phi$ as follows:

$$
\mathbf{b}_{M \times 1}=\phi_{M \times N} \mathbf{x}_{N \times 1}
$$

where $\boldsymbol{\phi}$ is measurement matrix. From (2) and (3) follows:

$$
\mathbf{b}=\phi \mathbf{x}=\phi \psi \mathbf{z}=\theta \mathbf{z}
$$

The system of equations defined by (4) consists of $M$ equations with $N$ unknowns, which implies infinite number of solutions $(M<<N)$. In order to obtain optimal solution, various minimization algorithms have been used. Each of them is based on finding the sparsest solution of the undetermined system of equations. Commonly used optimization technique is based on the $l_{1}$ - minimization [3]-[5], [16]:

$$
\hat{\mathbf{z}}=\min \|\mathbf{z}\|_{l_{1}} \text { subject to } \mathbf{b}=\boldsymbol{\theta} \mathbf{z},
$$

where $\hat{\mathbf{z}}$ is a solution of the minimization problem, whereas the $l_{1}$-norm of vector $\mathbf{z}$ is defined by [4], [5]:

$$
\|\mathbf{z}\|_{l_{1}}=\sum_{i=1}^{N}\left|z_{i}\right| \text {. }
$$

The problems in CS can be recast as linear programs or as second-order cone programs. Usually, the linear programming problems are solved using the basis pursuit primal-dual methods, while the second-order cone programs are solved using a generic log-barrier algorithm [5]. The $\ell_{1}$-norm based minimization problems can be solved using a variety of existing solvers such as: CVX, 11-magic, and LASSO.
ו1, $M E C O-2014$

Budva, Montenegro

\section{CS RECONSTRUCTION ALGORITHMS}

\section{A. Basis Pursuit}

Basis Pursuit [3]-[5], [17] is technique for finding signal representation in overcomplete dictionaries by convex optimization. Standard Basis Pursuit [3] algorithm is based on $\ell_{1}$ - minimization, and is described with equation (5). In the cases of compressible, but not exactly sparse $\mathbf{z}$, or $\mathbf{b}$ being corrupted by noise, equality constraints are modified. The $\ell_{1}$ regularization problem can be considered as quadratic programming as follows:

$$
\min _{z} \frac{1}{2}\|\mathbf{b}-\boldsymbol{\theta} \mathbf{z}\|_{2}^{2}+\lambda\|\mathbf{z}\|_{1}
$$

which is known as Basis Pursuit Denoising.

Basis Pursuit problem can be solved by using primal-dual interior point method. In the case of real $\mathbf{z}, \boldsymbol{\theta}$ and $\mathbf{b}$, problem (5) can be recast as [3]:

$$
\min _{p} \sum p, \text { s.t. }-p \leq z \leq p, \boldsymbol{\theta} \mathbf{z}=\mathbf{b},
$$

where variable $p$ is introduced to avoid absolute value in (6). Primal-dual procedure is defined with the following steps [4]:

1. For the known measurement vector $\mathbf{b}$, set $\mathbf{z}=\mathbf{z}_{\mathbf{0}}$ as

$$
\mathbf{z}_{0}=\theta^{\mathbf{T}} \mathbf{b} \text {. }
$$

2. Set $p_{0}=\alpha\left|\mathbf{z}_{\mathbf{0}}\right|+\beta \max \left\{\left|\mathbf{z}_{\mathbf{0}}\right|\right\}$, where $\alpha$ and $\beta$ are userdefined parameters.

3. Form the Lagrangian function as:

$$
\begin{aligned}
& L\left(z, p, v, \frac{-1}{z_{0}-p_{0}}, \frac{-1}{-z_{0}-p_{0}}\right)= \\
& =f(p)+v(\theta z-b)-\frac{z-p}{z_{0}-p_{0}}-\frac{(-z-p)}{-z_{0}-p_{0}}, \\
& \text { where } v=-\theta\left(\frac{-1}{z_{0}-p_{0}}+\frac{1}{-z_{0}-p_{0}}\right) .
\end{aligned}
$$

4. Each argument of the Lagrangian function is updated by step direction $(\Delta)$ and step length $(t)$. Step directions are obtained by finding first derivatives of $L$ in terms of its arguments. Step lengths are calculated using backtracking line search [5]. For example, a new value for $z$ is obtained as $z=z+t \Delta z$.

\section{B. Greedy Algorithms: Orthogonal Matching Pursuit and Iterative Hard Tresholding Algorithm}

Except by using optimization techniques, the sparsest solution could be obtained by using greedy search strategies [12], [13]. These strategies are much simpler and faster than, for example, $l_{1}$-norm based optimization techniques. Greedy methods are iterative procedures, which in each iteration select the element of the dictionary (transform matrix) that best matches the signal. In this paper Orthogonal Matching Pursuit 
$3^{\text {rd }}$ Mediterranean Conference on Embedded Computing

(OMP), [13], [18] and Iterative Hard Thresholding (IHT) [12] greedy methods will be described.

Knowing CS sensing matrix $\boldsymbol{\theta}$ and measurement vector $\mathbf{b}$ OMP approximate signal $\mathbf{z}$ as linear combination of columns in $\boldsymbol{\theta}$. In each iteration, a set of columns in expanded with additional column that best correlates with the residual signal. The algorithm terminates until residual falls below determined threshold. OMP [13] can be summarized as follows:

1. Variables initialization: set the approximation error $\mathbf{r}^{0}=$ $\mathbf{b}$, the initial solution to $\mathbf{z}^{0}=0$ and $\mathbf{S}^{0}=\varnothing$.

2. Do following steps till the stopping criterion is met:
a) $\mathbf{S}_{n}=\mathbf{S}_{n-1} \cup \arg _{i} \max \left|\left\langle\mathbf{r}_{n-1}, \boldsymbol{\theta}_{i}\right\rangle\right|$,
b) $\mathbf{z}_{n}=\arg \min _{z}\left\|\mathbf{r}_{n-1}-\mathbf{S}_{n} \mathbf{z}_{n-1}\right\|_{2}^{2}$,
c) $\mathbf{r}_{n}=\mathbf{r}_{n-1}-\mathbf{S}_{n} \mathbf{z}_{n-1}$.
d) $n=n+1 \quad$ and $\quad \mathbf{S}_{n}=\mathbf{S}_{n-1} \cup \arg _{i} \max \left|\left\langle\mathbf{r}_{n-1}, \boldsymbol{\theta}_{i}\right\rangle\right|$

until $n \leq K$, where $K$ is number of signal components.

IHT [12] is iterative algorithm which uses non-linear operator to reduce the $l_{0}$ - norm in each iteration, i.e.:

$$
\min _{z}\|\mathbf{b}-\boldsymbol{\theta z}\|_{2}^{2}+\lambda\|\mathbf{z}\|_{0} .
$$

From the optimization problem described by (11), the following iterative algorithm is derived. The non-linear operator is denoted as $H_{k}(a)$ and sets all but the largest (in terms of magnitude) $k$ elements of $a$ to zero. For given $\mathbf{z}^{0}$, algorithm iterate:

$$
\mathbf{z}^{n+1}=H_{k}\left(\mathbf{z}^{n}+\boldsymbol{\theta}^{T}\left(\mathbf{b}-\boldsymbol{\theta} \mathbf{z}^{n}\right)\right),
$$

until either $k>N$ max or $\left|\mathbf{b}-\boldsymbol{\theta} \mathbf{z}^{n}\right|_{2}<\mathcal{E}$. The $H_{k}$ is defined as follows:

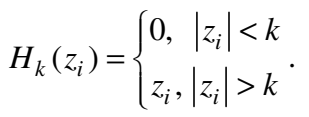

\section{ANALYSIS OF THE CS RECONSTRUCTION PERFORMANCE APPLIED TO SPARSE BAND-LIMITED SIGNALS}

In this section, two greedy algorithms - OMP and IHT, as well as two basis pursuit algorithms - standard BP and BP Denoising are applied on the sparse band-limited wireless signal. The CVX and Sparsify Matlab toolboxes are used for solving optimization problems. We assumed non-noisy and noisy signal case. Different SNRs are observed, as well. Signal is described as:

$$
x=A_{1} e^{j 2 \pi 34 n / N}+A_{2} e^{j 2 \pi 88 n / N}+A_{3} e^{j 2 \pi 128 n / N}+A_{4} e^{j 2 \pi 64 n / N},
$$

where component magnitudes are:

$A_{1}=4.5, A_{2}=4, A_{3}=2.5, A_{4}=4 ; N=512$ is length of the signal and $n \in(1, N)$. Signal consists of 4 components in the Fourier (DFT) domain and therefore is sparse in this domain. First the non-noisy case is observed. In all considered
ו'ו"1, MECO - 2014

Budva, Montenegro

algorithms, signal is reconstructed with 60 samples, which is $12 \%$ of the signal length. Fig. 1a shows time and Fig. 1b shows DFT domain of the original and reconstructed signals. Fig. 1a shows zoomed time region of the original and reconstructed signal on the same graph. All of the observed reconstruction algorithms give satisfactory results, with the mean square error (MSE) approximately the same. However, the reconstruction time is different for different algorithms.

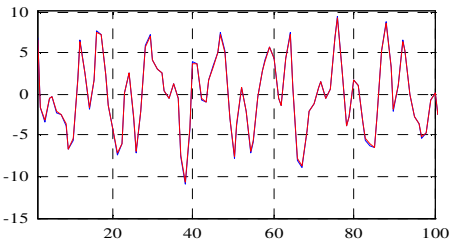

a)
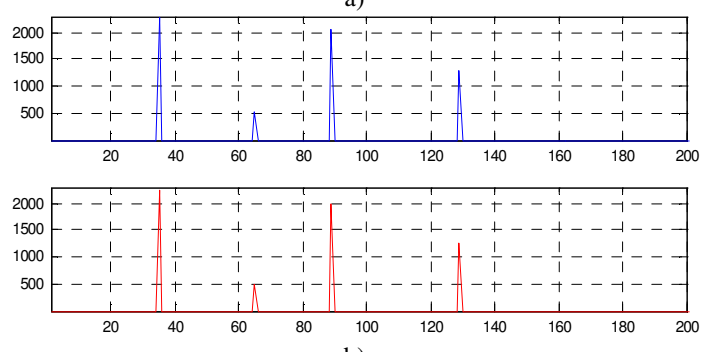

b)

Figure 1: a) Zoomed time domain of the original and reconstructed signal, b) zoomed Fourier domain of the original (blue) and reconstructed signal (red)

Table 1 shows MSEs and reconstruction times for the observed algorithms and for 60 samples taken as measurements in each case. From the Table 1 it can be seen that the IHT is the fastest, and BPD is the slowest among the observed algorithms. The results of the BPD algorithm are dependent on the parameter $\lambda$.

Table 1: Mean square errors and execution times for different algorithms

\begin{tabular}{|c|c|c|}
\hline \multirow{2}{*}{ ALGORITHM } & MSE & TIME (SEC) \\
\hline IHT & 0.0119 & 0.031599 \\
\hline OMP & 0.0119 & 0.050623 \\
\hline BP & 0.0119 & 2.390187 \\
\hline BPD & 0.012 & 4.773038 \\
\hline
\end{tabular}

Larger $\lambda$ causes the smaller magnitudes of the DFT components. OMP and IHT require exact number of signal components to be known, which could be limiting factor for their applications.

The performance of the observed optimization algorithms is also tested in the presence of Gaussian noise. Results related to the MSE for different SNRs and different algorithms are given in Table 2. The regularization parameter in BPD algorithm is chosen in a way that minimizes MSE. Larger values for 


\section{$3^{\text {rd }}$ Mediterranean Conference on Embedded Computing}

parameter $\lambda$ results in better noise reduction, and consequently, less number of peaks in the DFT domain. However, the larger $\lambda$ causes smaller signal components magnitudes in DFT domain, which increases the MSE between original and reconstructed signal and sometimes fails to reconstruct signal.

Table 2: Mean square errors for different SNRs and different algorithms

\begin{tabular}{|c|c|c|c|c|c|c|c|}
\hline $\begin{array}{c}\text { SNR } \\
\mathbf{1 8 . 0 7 9} \\
\text { DB }\end{array}$ & MSE & $\begin{array}{c}\text { SNR } \\
\mathbf{1 4 3 8}\end{array}$ & MSE & $\begin{array}{c}\text { SNR } \\
\mathbf{1 1 . 3 8 9} \\
\text { DB }\end{array}$ & MSE & $\begin{array}{c}\text { SNR } \\
\mathbf{8 . 9 7 5 9}\end{array}$ & MSE \\
\hline IHT & 0.0377 & IHT & 0.0941 & IHT & 0.1756 & IHT & FAILED \\
\hline OMP & 0.0377 & OMP & 0.0941 & OMP & 0.1756 & OMP & 0.1247 \\
\hline BP & 0.3059 & BP & 0.5425 & BP & FAILED & BP & FAILED \\
\hline BPD & 0.2326 & BPD & 0.3969 & BPD, & FAILED & BPD & FAILED \\
\hline
\end{tabular}

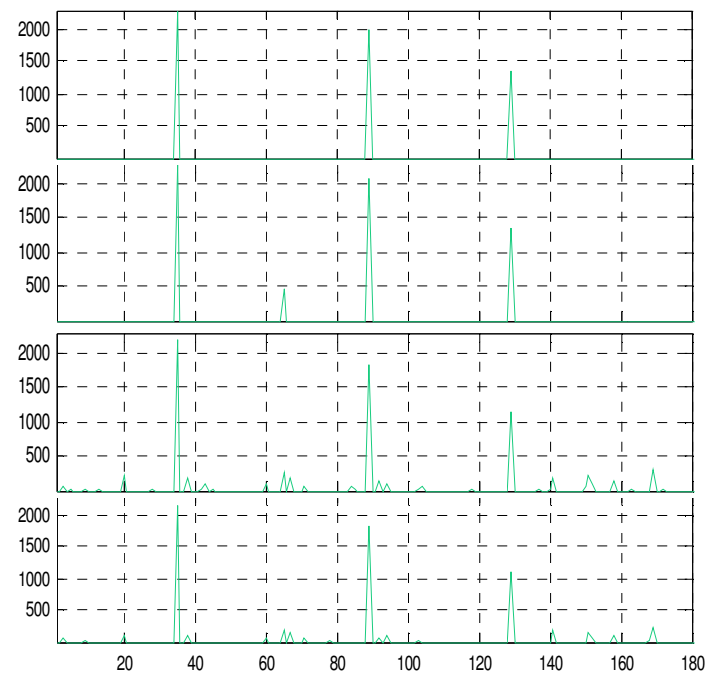

Figure 2: Reconstructed Fourier transform of the noisy signal $(\mathrm{SNR}=$ $8.9757 \mathrm{~dB}$ ), by using different reconstruction algorithms: IHT, OMP, BP and BPD, from top to the bottom

It is important to emphasize that execution time is the shortest for IHT and the longest for the BPD, as in the nonnoisy case. From the Table 2 it can be seen that OMP and IHT give the smallest MSEs among observed algorithms. In the case of $\mathrm{SNR}=8.9757 \mathrm{~dB}$ only the OMP succeeded to reconstruct signal exactly, as it can be seen form the Fig. 2. SNR is calculated as: $S N R=20 \log _{10}\left(A_{s} / A_{n}\right)$, where $A_{s}$ is signal amplitude and $A_{n}$ is noise amplitude. In the case of lower SNR, component with the smallest amplitude could not be detected.

\section{CONCLUSION}

Performance of the several reconstruction algorithms applied on sparse band-limited signal are considered in the paper. In all considered cases signal is reconstructed by using $12 \%$ samples of the total signal length. Algorithms are tested in the non-noisy and noisy cases. MSEs between original and reconstructed signal are measured, as well as algorithm execution time. It is shown that greedy algorithms give shorter execution time, and smaller MSE compared to the BP algorithms. Also, the best performance is obtained by using OMP, as it gives satisfactory reconstruction even if the SNR is $8.9759 \mathrm{~dB}$, when other considered algorithms failed in reconstruction. However, the exact number of signal components has to be a priori known in the case of greedy algorithms, which sometimes could be limiting factor.

\section{REFERENCES}

[1] L. Stankovic, S. Stankovic, M. Amin, "Missing Samples Analysis in Signals for Applications to L-estimation and Compressive Sensing," Signal Processing, vol. 94, Jan 2014, pp. 401-408, 2014.

[2] S. Stankovic, I. Orovic, M. Amin, "L-statistics based Modification of Reconstruction Algorithms for Compressive Sensing in the Presence of Impulse Noise," Signal Processing, vol.93, no.11, Nov. 2013.

[3] L. Stankovic, S. Stankovic, I. Orovic, M. Amin, "Robust TimeFrequency Analysis based on the L-estimation and Compressive Sensing," IEEE Signal Processing Letters, vol. 20, no. 5, pp. 499-502, 2013.

[4] E. Candes, J. Romberg, "11-magic: Recovery of Sparse Signals via Convex Programming", October 2005.

[5] S. Stankovic, I. Orovic, E. Sejdic, "Multimedia Signals and Systems," Springer-Verlag, New York, 2012.

[6] L. C. Potter, E. Ertin, J. T. Parker, M. Cetin, "Sparsity and Compressed Sensing in Radar Imaging," Proceedings of the IEEE, vol. 98, no.6, pp.1006-1020, June 2010.

[7] I. Orovic, S. Stankovic, "Compressive Sampling and Image Watermarking," ELMAR 2013, Zadar, Croatia, Sept. 2013.

[8] M. F. Duarte, R. G. Baraniuk, "Recovery of frequency-sparse signals from compressive measurements," 48th Annual Allerton Conference on Communication, Control, and Computing (Allerton), 2010, pp.599-606, Sept. 29-Oct. 12010.

[9] M. A. T. Figueiredo, R. D. Nowak,S. J. Wright, "Gradient Projection for Sparse Reconstruction: Application to Compressed Sensing and Other Inverse Problems," IEEE Journal of Selected Topics in Signal Processing, vol.1, no.4, pp.586-597, Dec. 2007.

[10] E. Sejdic, A. Can, L. F. Chaparro, C. M. Steele, T. Chau, "Compressive Sampling of Swallowing Accelerometry Signals Using Time-Frequency Dictionaries Based on Modulated Discrete Prolate Spheroidal Sequences", EURASIP Journal on advances in signal processing, 2012.

[11] S. Stankovic, I. Orovic, "Robust Complex-Time Distributions based on Reconstruction Algorithms," MECO - 2013, pp.105-108, June 2013, Budva, Montenegro, 2013.

[12] T. Blumensath, M. E. Davies, "Iterative Thresholding for Sparse Approximations", Journal of Fourier Analysis and Applications, vol. 14, no. 5-6, pp 629-654, December 2008.

[13] T. Blumensath, M. E. Davies, "Gradient Pursuits," IEEE Transactions on Signal Processing, vol.56, no.6, pp.2370-2382, June 2008.

[14] T. Roos, P. Myllymaki, J. Rissanen, "MDL Denoising Revisited," IEEE Transactions on Signal Processing, vol. 57, no.9, pp.3347-3360, Sept. 2009.

[15] I. Ramirez, G. Sapiro, "An MDL Framework for Sparse Coding and Dictionary Learning," IEEE Transactions on Signal Processing, vol. 60, no.6, pp.2913,2927, June 2012.

[16] S. Stankovic, I. Orovic, M. Amin, "Compressed Sensing Based Robust Time-Frequency Representation for Signals in Heavy-Tailed Noise," ISSPA 2012, Canada, 2012.

[17] P. R. Gill, A. Wang, A. Molnar, "The In-Crowd Algorithm for Fast Basis Pursuit Denoising," IEEE Transactions on Signal Processing, vol. 59, no. 10, pp. 4595-4605, Oct. 2011.

[18] S. Stankovic, I. Orovic, "An Ideal OMP based Complex-Time Distribution," MECO - 2013, pp. 109-112, June 2013, Budva, Montenegro, 2013. 